\title{
Electro-Optic Frequency Response Shaping in High Speed Mach-Zehnder Modulators
}

\author{
L. Breyne ${ }^{1,2}$, J. Lambrecht ${ }^{1}$, M. Verplaetse ${ }^{1}$, X. Yin ${ }^{1}$, G. Roelkens ${ }^{2}$, P. Ossieur ${ }^{1}$ and J. Bauwelinck ${ }^{1}$ \\ ${ }^{1}$ IDLab, INTEC, Ghent University - imec, 9052 Ghent, Belgium \\ ${ }^{2}$ Photonics Research Group, INTEC, Ghent University - imec, 9052 Ghent, Belgium \\ Laurens.Breyne@UGent.be
}

\begin{abstract}
We demonstrate a simple technique to shape the electro-optic frequency response of high-speed TW-MZMs. C-band transmission of $56 \mathrm{~Gb} / \mathrm{s}$ NRZ over $3 \mathrm{~km}$ SSMF shows $5 \mathrm{~dB}$ powerpenalty improvement at KP4-FEC between a standard and shaped MZM design.

OCIS codes: (060.2360) Fiber optics links and subsystems; (230.7020) Traveling-wave devices, (130.4110) Modulators
\end{abstract}

\section{Introduction}

Short-reach interconnects are under constant pressure to carry an increasing amount of data. This has led to a higher spectral link efficiency by using more complex modulation formats than NRZ and improvements in bandwidth of subsystems to push the baudrate. Focusing on the transmit side, significant effort is put into enhancing the bandwidth of the photonic modulator. For high-speed integrated Mach-Zehnder modulators (MZMs), silicon photonics is preferred over other material systems, like III-V or $\mathrm{LiNbO}_{3}$, due to the low cost, small footprint (compared to $\mathrm{LiNbO}_{3}$ ) high yield and potential high volume production. Higher bandwidths have been achieved by optimizing the PN junctions [1], local substrate removal [2], employing more exotic materials [3] and optimizing the transmission lines and electrodes [4]. Increasing the bandwidth has also been achieved by embedding equalization in the modulator design. For example [5] embeds passive RC equalization to boost the bandwidth of a lumped MZM. A more flexible solution is shown in [6]: a segmented MZM is used as optical-domain feedforward equalizer. Instead of only compensating the delay between segments due to velocity mismatch, the electrical signals for each segment are additionally delayed and scaled. This allows to make the trade-off between bandwidth and extinction ratio (ER). However, it requires a specific driver design and a large amount of connections between the driver and MZM.

We report a novel technique to perform electro-optic frequency response shaping in traveling wave (TW) MZMs. By using a dual-arm periodically loaded TW-MZM where optical delay and crossings are added between segments, the EO frequency response can be altered to extend the modulator bandwidth or induce peaking to overcome other bandwidth limitations in the optical link. We demonstrate performance improvement using a real-time $56 \mathrm{~Gb} / \mathrm{s} \mathrm{NRZ}$ (PRBS $2^{15}-1$ ) transmission experiment at $1550 \mathrm{~nm}$ by comparing a standard and a shaped TW-MZM. Over $3 \mathrm{~km}$ SSMF, the standard TW-MZM experiences a $5 \mathrm{~dB}$ power penalty at KP4-FEC compared to the shaped MZM.

\section{Electro-Optic Transfer Function Shaping in MZMs}

By periodically loading a transmission line with PN junctions, as analyzed e.g. in [4], lower losses and a better velocity matching can be achieved, leading to an increased bandwidth. An illustration of an MZM with 10 segments is shown in Fig. 1 (a). All phase shifters are equal and are spaced according to a fixed pitch. When a drive voltage $\mathrm{V}$ is applied to the input of the modulator, each segment induces a phase shift $\mathrm{PS}_{\mathrm{i}}$ between the upper and lower arm of the MZM. This phase shift can be calculated as $\mathrm{PS}_{\mathrm{i}}=\mathrm{PS}=\mathrm{V} \cdot \pi / \mathrm{V}_{\pi \mathrm{seg}}$, with $\mathrm{V}_{\pi \mathrm{seg}}$ the $\mathrm{V}_{\pi}$ of a single segment. When the voltage wave travels over the transmission line, it experiences a delay $\mathrm{T}_{\mathrm{E}}$ between subsequent segments. Similarly, the optical signal in the waveguide experiences a delay $\mathrm{T}_{\mathrm{O}}$ between adjacent segments. An equivalent block diagram describing the relationship between the voltage applied to the modulator and the phase shift induced in the MZM is given in Fig. 1 (b). Losses on the electrodes are neglected for now. To obtain maximum bandwidth, the modulator is designed such that the velocity of the electrical and optical signal are matched, so $T_{E}=T_{O}$. Now, the total phase shift is the sum of all individual phase shifts: $\mathrm{PS}_{\mathrm{tot}}=10 \mathrm{PS}=10 \mathrm{~V} \cdot \pi / \mathrm{V}_{\pi \mathrm{seg}}$.

In order to shape the electro-optic transfer function, we can add optical delay lines between adjacent segments or let the waveguides cross from one arm to the other, as shown for example in Fig. 1 (c). There is no interaction between the optical signals in a crossing, so the structure still behaves as one single MZM. Note that optical delays and crossings can be added between any pair of adjacent segments. The segments, PN junctions and electrodes remain untouched. This structure can be easily analyzed using the equivalent block diagram of Fig. 1 (d). The crossing between the $3^{\text {rd }}$ and $4^{\text {th }}$ segments does two things: the phase shifts induced by all segments before the crossing (against the propagation direction of the optical wave, so $\mathrm{PS}_{1}$ to $\mathrm{PS}_{3}$ ) are inverted and an additional waveguide is added so an additional delay $\mathrm{T}_{\mathrm{D}}$ is present in the optical domain. Between the $4^{\text {th }}$ and $5^{\text {th }}$ segments, an optical delay line with a delay $T_{D}$ is inserted. Since $T_{E}$ is still equal to $T_{O}$, the total phase shift at the output can now be written as $\mathrm{PS}_{\text {tot }}(\mathrm{t})=-\mathrm{PS}_{1}\left(\mathrm{t}-2 \mathrm{~T}_{\mathrm{D}}\right)-\mathrm{PS}_{2}\left(\mathrm{t}-2 \mathrm{~T}_{\mathrm{D}}\right)-\mathrm{PS}_{3}\left(\mathrm{t}-2 \mathrm{~T}_{\mathrm{D}}\right)+\mathrm{PS}_{4}\left(\mathrm{t}-\mathrm{T}_{\mathrm{D}}\right)+\mathrm{PS}_{5}(\mathrm{t})+\ldots+\mathrm{PS}_{10}(\mathrm{t})$. As all segments are the same, we can 
also write: $\mathrm{PS}_{\text {tot }}(\mathrm{t})=-3 \mathrm{PS}\left(\mathrm{t}-2 \mathrm{~T}_{\mathrm{D}}\right)+\mathrm{PS}\left(\mathrm{t}-\mathrm{T}_{\mathrm{D}}\right)+6 \mathrm{PS}(\mathrm{t})$. Converting to the $\mathrm{Z}$-domain yields the following $2^{\text {nd }}$ order filter: $\mathrm{PS}_{\text {tot }}(\mathrm{z})=\left(-3 \mathrm{z}^{-2}+\mathrm{z}^{-1}+6\right) \mathrm{PS}(\mathrm{z})=\left(-3 \mathrm{z}^{-2}+\mathrm{z}^{-1}+6\right) \mathrm{V}(\mathrm{z}) \cdot \pi / \mathrm{V}_{\text {sseg. }}$. With $\mathrm{z}^{-1}=\exp \left(-\mathrm{j} \pi 2 \mathrm{f} / \mathrm{f}_{\mathrm{s}}\right)$ and $\mathrm{f}_{\mathrm{s}}=1 / \mathrm{T}_{\mathrm{D}}$. At $\mathrm{DC}, \mathrm{PS}_{\text {tot }}$ is only 4PS compared to 10PS for the standard MZM. But this response induces $7 \mathrm{~dB}$ peaking. This will drop when transmission line loss is included. The peaking frequency can be adjusted by varying $T_{D}$.

As optical delay lines and crossings can be placed between any pair of segments, different transfer functions can be realized. This allows a significant increase in modulator bandwidth or added peaking in the response to alleviate bandwidth limitations in the link. The simulation in Fig. 1 (e), with the MZM layout described in Section 3, shows the $\mathrm{EO}$ bandwidth as a function of $\mathrm{V}_{\pi}$ for the standard periodically loaded MZM with 4, 6, 8 and 10 segments (blue). The red curve shows the EO bandwidth as a function of $\mathrm{V}_{\pi}$ for a shaped MZM with 10 segments where various configurations of optical delay lines and crossings are used to boost the bandwidth. It is clear that for the same $\mathrm{V}_{\pi}$, larger bandwidths can be obtained using our proposed technique. For very long MZMs, the electrode losses may dominate over the shaping thus limiting the bandwidth enhancement.

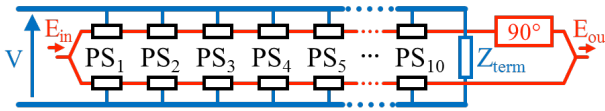

(a)

(b)

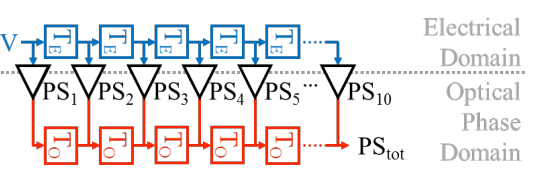

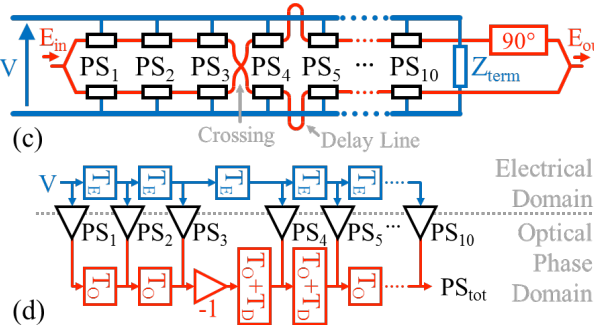

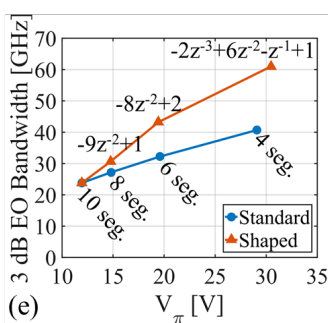

Fig. 1: (a) Standard 10-segment periodically loaded MZM; (b) equivalent block diagram of (a); (c) 10 segment periodically loaded MZM with transfer function shaping (d); equivalent block diagram of (c); (e): Simulation of standard MZM with 4, 6, 8 or 10 segments vs. various configurations of a shaped MZM with 10 segments. Note: the simulations accuracy decreases above $40 \mathrm{GHz}$.

\section{Design and characterization}

The modulators from Fig. 1(a) and (c) have been fabricated on imec's iSiPP50G silicon photonic platform [7]. Both are implemented in order to compare the effect of shaping. The design of Fig. 1 (c) is chosen as the peaking can be used to compensate for the bandwidth deterioration due to a chromatic dispersive channel. The layout of the shaped MZM is shown in Fig. 2 (a), the insets show how a crossing and delay are implemented in optical waveguides. The 10 segments are each 175 um long and have a pitch of $250 \mathrm{um}$. The PN junctions have the same dimensions as those used for the default iSiPP50G phase shifter. The modulator is $2.5 \mathrm{~mm}$ long. As we target $56 \mathrm{~Gb} / \mathrm{s}$ transmission, the delay is optimized using simulations to have maximal peaking at $25-30 \mathrm{GHz}$. The optimum delay is $7 \mathrm{ps}$, resulting in a 500 um delay line. A differential GSSG electrode configuration is used. The depletion PN junction phase shifters are placed in series with the signal lines and are biased through a thin, long inductive line. A segment cross section is shown in Fig. 2 (b). Termination resistors are present on-chip, a thermo-optic heater is used to bias the MZM at quadrature. The standard MZM uses exactly the same design but with direct connections between all segments.
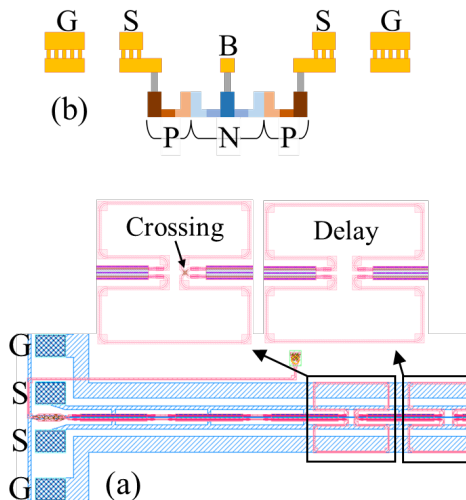

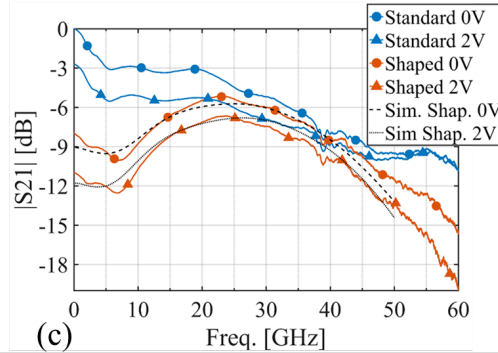

(c)

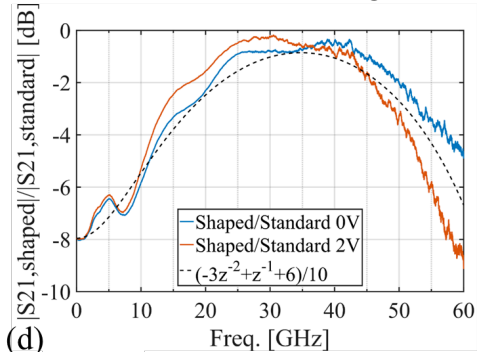

(d)

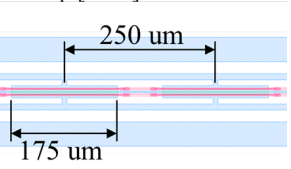

Fig. 2: (a) Layout of the MZM with shaping, insets of the crossing, delay and segments; (b) cross section of the transmission line at a segment; (c) simulated and measured electro-optic transfer function (d) measured effect of the transfer function shaping compared to the theoretical shape.

Measurements show that at DC, the $\mathrm{V}_{\pi}$ of the standard and shaped modulator is respectively $11.8 \mathrm{~V}$ and $29.6 \mathrm{~V}$ (PN reverse bias $1 \mathrm{~V}$ ). As explained earlier, this is because only 4 of the 10 segments are actually contributing to the DC phase shift. The insertion loss at a reverse bias of $1 \mathrm{~V}$ coming from the PN junctions is in both designs very similar, $2.6 \mathrm{~dB}$ and $3.1 \mathrm{~dB}$ for the standard and shaped modulator respectively. The small deviation is caused by $0.3 \mathrm{~dB}$ loss from the crossing and 2 times $0.1 \mathrm{~dB}$ from the additional waveguide. The transfer functions were measured using a 
vector network analyzer and a $70 \mathrm{GHz}$ photodiode, the results are shown in Fig. 2 (c). The $3 \mathrm{~dB}$ bandwidth of the standard modulator is 21 and $25.1 \mathrm{GHz}$ at 0 and $2 \mathrm{~V}$. For the shaped modulator, there is $3.2 \mathrm{~dB}$ peaking at $23.2 \mathrm{GHz}$ for $0 \mathrm{~V}$ reverse bias, which increases to $4.6 \mathrm{~dB}$ at $23.8 \mathrm{GHz}$ for a reverse bias of $2 \mathrm{~V}$. The change in junction capacitance changes the transmission line characteristics resulting in more or less peaking. The reference amplitude is chosen at $1 \mathrm{GHz}$. Our simulations of the shaped modulator (valid up to $40 \mathrm{GHz}$ ) show good similarity with the measurements. By dividing the transfer functions of the shaped and standard modulator, the electrical effects can be eliminated and the effect of the transfer function shaping can be investigated. Fig. 2 (d) shows the measurement result is very close to the theoretical transfer function derived earlier.

\section{Measurements and results}

A transmission experiment at $56 \mathrm{~Gb} / \mathrm{s} \mathrm{NRZ} \mathrm{(PRBS} 2^{15}-1$ ) was conducted to analyze the performance improvement of the shaped design. The experimental setup is shown in Fig. 3 (a). The laser generates $13 \mathrm{dBm}$ at $1550 \mathrm{~nm}$. A polarization controller is used before light is sent to the polarization sensitive grating coupler through the fiber probe. The MZM-under-test is driven by the arbitrary waveguide generator (AWG) and $2 \times 24 \mathrm{~dB}$ amplifiers to obtain $4 \mathrm{~V}_{\text {ppdiff }}$ at the input of the MZM. The TX power launched into the fiber is around $1 \mathrm{dBm}$. The $12 \mathrm{~dB}$ insertion loss of the TX consists of $2 \times 3 \mathrm{~dB}$ from the grating couplers, $3 \mathrm{~dB}$ from the phase shifters and $3 \mathrm{~dB}$ because the MZM is biased at quadrature. To optimize the ER, the PN junctions are reverse biased at $0.5 \mathrm{~V}$. The modulated optical signal is sent through 0,2 or $3 \mathrm{~km} \mathrm{SSMF.} \mathrm{A} \mathrm{VOA} \mathrm{is} \mathrm{placed} \mathrm{to} \mathrm{control} \mathrm{the} \mathrm{light} \mathrm{entering} \mathrm{the} \mathrm{EDFA.} \mathrm{The}$ EDFA, subsequent optical filter, VOA and $70 \mathrm{GHz}$ photodiode compose a reference receiver. The EDFA helps to increase the RX sensitivity as no TIA is used. The optical filter is $1.2 \mathrm{~nm}$ wide and is used to suppress the ASE-ASE beating noise from the EDFA. The output of the PD is connected to a sampling scope to observe the eye diagrams or to an $11 \mathrm{~dB}$ amplifier and a DEMUX to create two $28 \mathrm{~Gb} / \mathrm{s}$ streams of which one is analyzed by the BER tester.
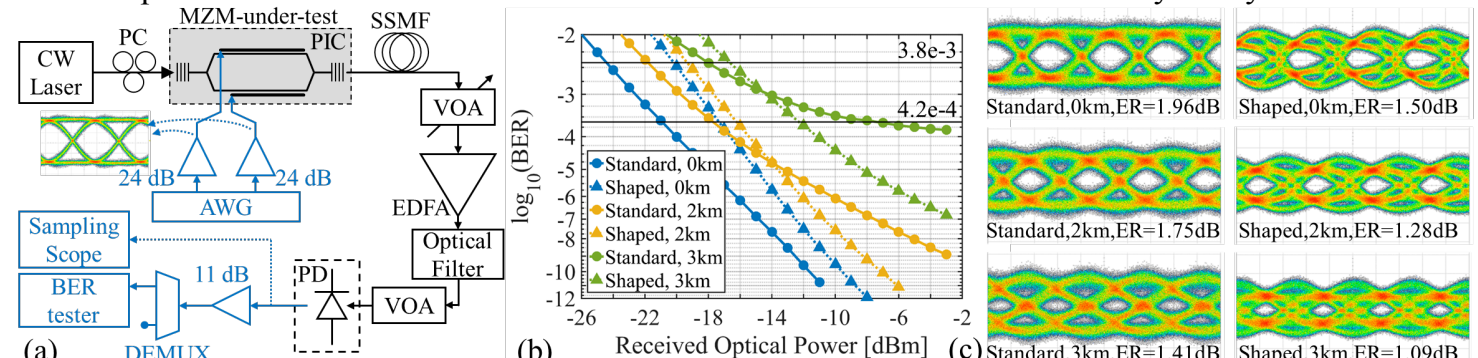

(b) Received Optical Power [dBm] (c) Standard,3km,ER=1.41dB Shaped,3km,ER=1.09dB

Fig. 3: (a) experimental setup: PC: polarization controller, VOA: variable optical attenuator; (b) BER curves for the standard and shaped MZM for $56 \mathrm{~Gb} / \mathrm{s}$ over 0,2 and $3 \mathrm{~km}$ as a function of power incident on the EDFA; (c) eye diagrams of the standard and shaped MZM for $56 \mathrm{~Gb} / \mathrm{s}$.

The measured BERs are shown in Fig. 3 (b) as a function of power incident to the EFDA. At 0 km, the penalty in modulation efficiency between the standard and shaped MZM is clearly visible as a power penalty of $4 \mathrm{~dB}$ at $7 \%$ OH HD-FEC (pre-FEC BER: 3.8e-3) and $3.5 \mathrm{~dB}$ at KP4-FEC (pre-FEC BER: 4.2e-4). At $2 \mathrm{~km}$, the shaped MZM is $2.5 \mathrm{~dB}$ and $1.5 \mathrm{~dB}$ worse at HD- and KP4-FEC than the standard design. However, at higher powers, the shaped modulator can achieve a BER $<1 \mathrm{e}-12$, which is not possible with the standard MZM. At $3 \mathrm{~km}$, the shaped modulator is $1.5 \mathrm{~dB}$ worse at HD-FEC, but $5 \mathrm{~dB}$ better at KP4-FEC. At the maximum power in the EDFA, $-3 \mathrm{dBm}$ limited by the insertion loss of the fiber and VOA, the BER of the shaped modulator is 3 decades better than the standard design. Further tuning of the transfer function used for the shaped frequency response may improve the ER while keeping enough peaking to counteract the dispersive link. In Fig. 3 (c), the various eyes at the PD output are shown.

\section{Conclusion}

We have demonstrated a novel technique to perform the shaping of the electro-optic transfer function of periodically loaded traveling wave MZMs. Only optical waveguides were modified, all other parts of the MZM remain untouched, no process modifications are required. The technique is applied to a silicon photonic MZM where peaking is introduced to counteract chromatic dispersion. C-band transmission of $56 \mathrm{~Gb} / \mathrm{s} \mathrm{NRZ} \mathrm{(PRBS} 2^{15}-1$ ) over $2 \mathrm{~km}$ yields a BER $<1 \mathrm{e}-12$ compared to $1 \mathrm{e}-9$ for a standard MZM without shaping. Transmission over $3 \mathrm{~km}$ yields $5 \mathrm{~dB}$ improvement in achieved optical budget at KP4-FEC. The proposed technique shows promising results to enhance the bandwidth of MZMs and to tailor MZM transfer functions to a range of desired responses.

This work was supported by the UGent Special Research Fund (BOF) and the ERC Advanced Grant 695495 (ATTO)

\section{References}

[1] D. Petousi et al, JQSTE, vol.21, no.4, pp.199-206, 2015

[2] M. Li et al, Photon. Res., vol.6, no.2, pp.109-116, 2018

[3] H. Zwickel et al, Opt. Expr., vol.25, no.20, pp.23784-23800, 2017

[5] S. Tanaka et al, JLT, vol.36, no.5, pp.1275-1280, 2018

[4] D. Patel, Opt. Expr., vol.23, no.11, pp.14263-14287, 2015

[6] B. Lee, JLT, vol. 37, no. 1, pp.89-94, 2019

[7] B. Snyder, OFC, San Diego, CA, 2018, Tu2A.3 